\title{
Die Beurteilung der Arbeitsfähigkeit ist und bleibt eine ärztliche Kernkompetenz!
}

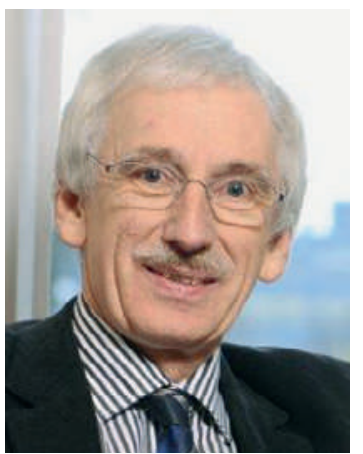

Das Bundesgerichtsurteil vom 12. Juni 2014 «Keine Korrelation Diagnose - Arbeitsunfähigkeit» hat in den Medien hohe Wellen geworfen: Wer hat nun das Sagen, die Ärztin oder der Jurist? Die im Urteil erwähnten Grundsätze sind seit längerem bekannt und entsprechen der aktuellen Rechtsprechung. So ist es Sache des Arztes, den Gesundheitszustand zu beschreiben und zu beurteilen. Bei der Folgenabschätzung bezüglich einer IV-Rente hat die ärztliche Person aber keine abschliessende Beurteilungskompetenz, hier müssen «Medizin» und «Recht» zusammenwirken. Persönlich bin ich über diese Interaktion gerade bei gewissen Rentenentscheidungen keinesfalls unglücklich! Dieses Bundesgerichtsurteil dokumentiert aber klar die wichtige Rolle der Ärztin respektive des Arztes rund um die Arbeitsfähigkeit. Der Zeugniswunsch, der nur allzu oft bei der Verabschiedung auf der Schwelle der Praxistüre stattfindet, darf keinesfalls dazu führen, dass die Beurteilung und die exakte Dokumentation darunter leiden. Eine eingeschränkte Arbeitsfähigkeit ist exakt zu protokollieren und zu begründen und soll keinesfalls pauschalisierend sein. Auf dem Zeugnis sind die Arbeitszeit und die Arbeitsleistung klar zu vermerken, ebenso das Datum der nächsten Konsultation. Rückwirkende Zeugnisse dürfen höchstens in ausgewählten, klar begründeten Ausnahmefällen ausgestellt werden. Ein allfällig anderweitiger Einsatz speziell bei längerer Einschränkung der Arbeitsfähigkeit darf nicht vergessen werden. Wichtig ist gerade dann ein Gespräch mit dem Arbeitgeber bzw. dem Personalchef selbstverständlich nur in Absprache mit der Patientin oder dem Patienten und wenn die entsprechende Einwilligung vorliegt.

\section{Medizin und Recht sind bei der}

\section{Abschätzung einer IV-Rente gefragt. Die} Beurteilung des Gesundheitszustandes obliegt allein der Ärztin bzw. dem Arzt.

Wichtig ist in der Arbeitsfähigkeitsbeurteilung, dass die nachfolgenden Faktoren nicht zu einer Fehlbeurteilung führen. Nicht versicherte Leistungen sind zum Beispiel Alter, Wirtschaftslage, Situation auf dem Stellenmarkt, Arbeitslosigkeit, soziokulturelle Faktoren, familiäre Verhältnisse, Bildungsstand, Sprache, Ethnie, Religion, Motivation und Aggravation.
Das Ziel soll einerseits eine optimale Rekonvaleszenz, andererseits aber auch eine rasche Wiedereingliederung der Patienten in den Arbeitsprozess sein. Denn je länger eine Arbeitsunfähigkeit dauert, desto grösser wird das Risiko für einen Verlust der Arbeitsstelle und desto mehr wächst das Rentenrisiko. Wer mehr als sechs Monate krankheitsbedingt nicht mehr im Arbeitsprozess war, hat ein Rentenrisiko von über $50 \%$ und nach einem Jahr sinken die Chancen für eine Reintegration auf gegen $0 \%$.

Dass eine Optimierung der Arbeitsfähigkeitsproblematik unter voller Wahrung der Interessen der betroffenen Patienten keine Utopie ist, wurde in einem beispielhaften Projekt im Kanton St.Gallen mit dem neuen Arztzeugnis gezeigt. Zentral hat sich dabei die verbesserte Kommunikation der Beteiligten erwiesen sowie ein Zusammenarbeitscredo. Dieses beinhaltet nebst der obigen optimalen Rekonvaleszenz und raschen Eingliederung die folgenden Zielsetzungen:

\section{Eine verbesserte Kommunikation sowie ein Commitment der Zusammenarbeit sind wegweisend bei der Beurteilung der Arbeitsfähigkeit.}

- Prioritär sind die Gesundheit der Arbeitnehmenden sowie der Erhalt des Arbeitsplatzes.

- Durch koordinierte Zusammenarbeit sollen Arbeitsausfälle, aber auch Gesundheitskosten reduziert werden.

Gerne verweise ich hierzu auch auf die Homepage der Ärztegesellschaft des Kantons St. Gallen: www.aerzte-sg.ch - Informationen - Absenzenmanagement.

Der Beitrag der Medizin für den Wirtschaftsstandort Schweiz ist enorm und wichtig. Im Jahr 2012 gingen in den hiesigen Betrieben gemäss Bundesamt für Statistik über 180000000 Arbeitsstunden wegen Krankheit oder Unfall verloren. Mit einem guten Präsenz- und Gesundheitsmanagement konnten die krankheitsbedingten Arbeitsunfähigkeitszeiten im Rahmen unseres Projektes um 30-50\% auf gegen $3 \%$ der Gesamtarbeitszeit reduziert werden. (PS: Im staatlichen Gesundheitswesen in England liegt dieser Wert bei über $10 \%$ !)

Ihre engagierte ärztliche Tätigkeit führt somit nicht nur zu einer besseren Volksgesundheit, sondern auch zu einem grossen volkswirtschaftlichen Nutzen - herzlichen Dank!

Dr. med. Peter Wiedersheim, Präsident der FMH-Delegiertenversammlung 